\title{
BIOCHEMICAL CHANGES OCCURING IN NEONATES WITH SEPSIS
}

\author{
Irina-Bianca KOSOVSKI ${ }^{1}$, Dana-Valentina GHIGA ${ }^{2 *}$, Cristina Nicoleta CIUREA ${ }^{3}$, \\ Anca BACÂREA ${ }^{4}$
}

\begin{abstract}
${ }^{1}$ Clinical Laboratory of County Emergency Clinical Hospital of Tîrgu Mureș, Romania
${ }^{2}$ Department of Research methodology, University of Medicine, Pharmacy, Science and Technology of Tîrgu Mureș, Romania

${ }^{3}$ Department of Microbiology, Virology, Parasitology, University of Medicine, Pharmacy, Science and Technology of Tîrgu Mureș, Romania

${ }^{4}$ Department of Pathophysiology, University of Medicine, Pharmacy, Science and Technology of Tîrgu Mureș, Romania
\end{abstract}

\author{
*Correspondence: \\ Dana-Valentina GHIGA \\ dana.ghiga@umfst.ro
}

Received: 20 June 2019; Accepted: 25 June 2019; Published: 30 June 2019

\begin{abstract}
This retrospective study aims to analyze the relationship between biochemical changes occurring in newborns with sepsis proven by positive blood culture (BC) and possible correlations with 1 min Apgar score, 5 min Apgar score, gestational age (GA), and birth weight (BW). We included in the study all infants $\leq 7$ days of life with positive BC that were admitted to the Neonatal Intensive Care Unit (NICU) and Neonatology Department (ND) of the County Emergency Clinical Hospital of Târgu Mureş, a tertiary level hospital, between 2014-2018. The analyzed parameters are: day of life for blood sampling (0-7 days of life), gender, Apgar score (1 and 5 minute), GA, BW, urea, creatinine, total bilirubin, direct bilirubin, aspartate aminotransferase (AST/GOT), alanine aminotransferase (ALT/GPT), c-reactive protein (CPR), bacteria involved, empiric antibiotics administered before blood sampling, temperature of the newborn on the day of BC. We found there is a statistically significant negative correlation between 1 and $5 \mathrm{~min}$ Apgar score and creatinine, between GA and urea and also between BW, GA and Direct Bilirubin. We found a statistically significant positive correlation between BW, GA and GPT.
\end{abstract}

Keywords: neonate, septicemia, bacteremia, biochemistry, CRP.

\section{Introduction}

According to World Health Organization (WHO) in 2017, globally 2.5 million children died in the first month of life, approximately 7000 newborn deaths every day with about 1 million dying on the first day and close to 1 million dying within the next 6 days.

The most common causes are: preterm birth, intrapartum-related complications (birth asphyxia, lack of breathing at birth), infections and birth defects. The vast majority of newborn deaths take place in low and middle-income countries (WHO, 2018). Annual neonatal mortality rates (NMRs, the probability of dying during the first 28 days of life) vary widely across the world, but west and Central Africa and South Asia had the highest NMRs while Western Europe has the lowest NMRs in 2017 (Hug et al., 2019). 
Neonatal sepsis is defined as a systemic infection (positive culture of blood, urine or cerebrospinal fluid) occurring in infants at $\leq 28$ days of life. According to the time of onset of the disease, neonatal sepsis may be classified in early onset (EOS, defined as a positive culture during $\leq 3$ days of life) and late onset (LOS, a positive culture $>3$ days of life) (Simonsen et al., 2014).

\section{Materials and Methods}

The study was approved by the Ethics Committee of the County Emergency Clinical Hospital of Târgu Mureş and it follows the Helsinki Declaration principles.

A 5 years retrospective study, from 20142018, was performed in June 2019, to evaluate the relationship between biochemical changes occurring in newborns with sepsis proven by positive blood culture (BC) and possible correlations with 1 min Apgar score, 5 min Apgar score, gestational age (GA), birth weight (BW).

Data were collected of all infants $\leq 7$ days of life with positive BC that were admitted to the Neonatal Intensive Care Unit (NICU) and Neonatology Department (ND) of the County Emergency Clinical Hospital of Târgu Mureş, a tertiary level hospital.

The data were obtained by accessing the H3 electronic medical database and the laboratory records. Tracked parameters:

- general data: day of life that the blood sampling was performed (0-7 days of life), gender, Apgar score (1 and 5 minute), GA, BW

- biochemical data: urea, creatinine, total bilirubin, direct bilirubin, aspartate aminotransferase (AST/GOT), alanine aminotransferase (ALT/GPT), c-reactive protein (CPR)

- microbiological data: positive $\mathrm{BC}$, bacteria involved, empiric antibiotics administered before blood sampling, temperature at blood sampling for BC.
We set a maximum of 2 days between the biochemical analyses and the date of blood sampling for the BC.

Patients excluded:

- Microorganism considered to be contaminants: Methylobacterium spp., Streptococcus mitis, Streptococcus oralis, Micrococcus luteus, Ochrobactrum anthropi and the association over 3 types of germs;

- A positive BC with coagulase-negative staphylococci (CoNS) and when in the comments of the microbiologist it was specified contamination, possible contamination or skin flora.

The data analysis included descriptive statistics elements (frequency, percentage, confidence interval 95\%, mean, median, standard deviation) and inferential statistics. The D'Agostino \& Pearson test was applied to determine the distribution of the analyzed data series. The Pearson correlation coefficient, respectively Spearman, was calculated. The significance threshold chosen for $p$ was 0.05 . The statistical analysis was performed using the GraphPad Prism 7 utility, the Trial variant.

\section{Results and discussions}

We identified $694 \mathrm{BC}$ performed on first 7 days of life on neonates, which of 88 (12.68\%) are positive, $26(3.74 \%)$ contaminated and 62 $(8.94 \%)$ true positive BC. In our group 24 $(38.70 \%)$ neonates are from the NICU and 38 $(61.30 \%)$ are from the ND. $13(20.96 \%)$ of newborns with sepsis died in hospital. Table 1 contains the description of the studied group.

In our group, $32(51.61 \%)$ of neonates had EOS and 30 (48.39\%) had LOS. The bacteria being identified and the antibiotics used, if it were the case, are presented in Table 2 . The most common empirical choices of antibiotics for the treatment of neonatal sepsis were Aminoglycoside (in EOS 5, 71.42\%; in LOS 7, $22.58 \%$ ) and Penicillin (in EOS 4, 57.14\%; in LOS $12,38.70 \%$ ) and for both categories. 
Table 1. The description of the studied group

Parameter (unit of measure, number of values

$M e a n \pm S D^{a}$

Median

Normal range $e^{e}$

\begin{tabular}{|c|c|c|c|}
\hline Age (days, 62) & $3.5 \pm 2.31$ & 3 & \\
\hline Gestational age (weeks, 62) & $35.13 \pm 4.44$ & 36 & \\
\hline Birth weight (grams, 62) & $2482 \pm 1050$ & 2530 & \\
\hline $1 \min A P G A R$ score (62) & $7.22 \pm 2.28$ & 8 & \\
\hline $5 \min A P G A R$ score $(62)$ & $8.06 \pm 1.83$ & 9 & \\
\hline Temperature at blood collection $\left({ }^{\circ} \mathrm{C}, 62\right)$ & $36.98 \pm 0.65$ & 37 & \\
\hline Urea $(m g / d L, 47)$ & $56.25 \pm 39.37$ & 43.98 & $9-14$ \\
\hline Creatinine $(\mathrm{mg} / \mathrm{dL}, 50)$ & $0.78 \pm 0.39$ & 0.66 & $0.17-0.85$ \\
\hline Total Bilirubin (mg/dL, 48) & $7.24 \pm 6.08$ & 6.11 & $0-12.6$ \\
\hline Direct Bilirubin $(m g / d L, 43)$ & $0.80 \pm 0.53$ & 0.57 & $0-0.6$ \\
\hline$G O T^{b}(U / L, 52)$ & $60.97 \pm 48.48$ & 48 & $0-110$ \\
\hline$G^{\prime} P^{c}(U / L, 50)$ & $35.34 \pm 48.96$ & 16 & $0-60$ \\
\hline$C R P^{d}(m g / L, 50)$ & $82.10 \pm 92.98$ & 53.71 & $0-5$ \\
\hline
\end{tabular}

Note: a - Standard Deviation; b - aspartate aminotransferase; c - alanine aminotransferase; $\mathrm{d}$ - c-reactive protein; e - normal clinical biochemistry reference ranges for neonates in Clinical Laboratory of County Emergency Clinical Hospital of Târgu Mureş (data from the manufacturer and the literature)

Table 2. The bacteria identified and the antibiotics used

\begin{tabular}{|c|c|c|c|c|}
\hline Parameter & & Frequency & Percentage & $\begin{array}{c}\text { Confidence interval } \\
(95 \%)\end{array}$ \\
\hline \multirow[t]{2}{*}{ Gender } & Female & 23 & $37.10 \%$ & $25.16 \%-50.31 \%$ \\
\hline & Male & 39 & $62.90 \%$ & $49.69 \%-74.84 \%$ \\
\hline \multirow[t]{10}{*}{ Positive bacteria } & Streptococcus & 3 & $4.84 \%$ & $1.01 \%-13.50 \%$ \\
\hline & Staphylococcus & 20 & $32.26 \%$ & $20.94 \%-45.34 \%$ \\
\hline & Stenotrophomonas maltophilia & 2 & $3.23 \%$ & $0.39 \%-11.17 \%$ \\
\hline & Escherichia coli & 10 & $16.13 \%$ & $8.02 \%-27.67 \%$ \\
\hline & Enterococcus & 9 & $14.52 \%$ & $6.86 \%-25.78 \%$ \\
\hline & Listeria & 2 & $3.23 \%$ & $0.39 \%-11.17 \%$ \\
\hline & Klebsiella & 7 & $11.29 \%$ & $4.66 \%-21.89 \%$ \\
\hline & Candida & 7 & $11.29 \%$ & $4.66 \%-21.89 \%$ \\
\hline & Serratia & 2 & $3.23 \%$ & $0.39 \%-11.17 \%$ \\
\hline & Acinetobacter & 2 & $3.23 \%$ & $0.39 \%-11.17 \%$ \\
\hline \multirow{6}{*}{$\begin{array}{l}\text { Empiric } \\
\text { antibiotics } \\
\text { administered } \\
\text { before blood } \\
\text { sampling }\end{array}$} & Aminoglycoside & 12 & $50.00 \%$ & $29.12 \%-70.88 \%$ \\
\hline & Penicillin & 16 & $66.67 \%$ & $44.68 \%-84.37 \%$ \\
\hline & Carbapenem & 5 & $20.83 \%$ & $7.13 \%-42.15 \%$ \\
\hline & Cephalosporin & 3 & $12.50 \%$ & $2.66 \%-32.36 \%$ \\
\hline & Polymyxin & 4 & $16.67 \%$ & $4.74 \%-37.38 \%$ \\
\hline & Fluoroquinolone & 1 & $4.17 \%$ & $0.11 \%-21.12 \%$ \\
\hline \multirow{2}{*}{$\begin{array}{l}\text { Blood sampling } \\
\text { on treatment }\end{array}$} & No & 38 & $61.29 \%$ & $48.07 \%-73.40 \%$ \\
\hline & Yes & 24 & $38.71 \%$ & $26.60 \%-51.93 \%$ \\
\hline
\end{tabular}


Of the 24 newborns receiving antibiotic empirical therapy, $16(66.66 \%)$ of them had associations of drug classes (14, 58.33\% received association with 2 classes and 2, $8.33 \%$ association with 3 classes). The most common association of drug classes was Penicillin with Aminoglycosides (7, 43.75\%).

In Table 3 correlations between $1 \mathrm{~min}$ Apgar score, 5 min Apgar score, GA, BW and biochemical parameters are presented.

Acute kidney failure (AKF) is a common clinical problem in NICUs. According to Mathur et al. (2006), in India, renal failure occurred in $26 \%$ neonates with sepsis and Low birth weight is an important risk factor for the development of AKF, a significantly higher number of babies with AKF weighed less than $2500 \mathrm{gm}$. The mortality was three times higher in neonates with AKF. In Turkey, Agras et al. (2004) found a frequency of $3.4 \%$ AKF in the NICU, the premature newborns constituting $31.1 \%$ of the cases. The most common condition that contributed to AKF that they found was asphyxia (40.0\%) followed by sepsis/metabolic disease $(22.2 \%)$ and feeding problems (17.8\%). In another study, also conducted in Turkey, the prevalence of neonatal AKF was $8.4 \%$. The common cause of AKI was respiratory distress syndrome, followed by sepsis, asphyxia, dehydration, congenital anomalies of the urinary tract, congenital heart disease, and medication. In that case, the overall mortality rate was $23.8 \%$ (Bolat et al., 2013). In Egypt, $40.7 \%$ of the AKI cases were born after full-term pregnancy while $59.3 \%$ were pre-term babies. The predisposing factors for AKI were sepsis (63\%), respiratory distress syndrome $(55.6 \%)$, mechanical ventilation $(51.9 \%)$, peri-natal asphyxia (18.5\%), dehydration (14.8\%), surgical operation $(11.1 \%)$, congenital heart disease $(7.4 \%)$, sub-galeal hematoma $(3.7 \%)$, polycythemia $(3.7 \%)$ and intra-ventricular hemorrhage (3.7\%) (Youssef et al., 2015). Although the prevalence and mortality rate are different depending on the hospital, the causes remain roughly the same and sepsis is found everywhere. We found a statistically significant negative correlation between a high 1 and 5 min Apgar score and a low value of creatinine. We have also found a statistically significant negative correlation between a high GA and a low value of urea.

Hepatic pathology is common among newborns with sepsis. Jaundice is a wellknown complication of sepsis or nonbacterial infection. Sepsis and bacterial infection are responsible for up to $20 \%$ of cases of jaundice in patients of all ages in a community hospital setting (Whitehead et al., 2001). Sepsis is more likely to manifest with jaundice in infants and children than in adults. Various mechanisms that can lead to hyperbilirubinemia alone during systemic infection are hemolysis, hepatic dysfunction, cholestasis (Chand and Sanyal, 2007). We found that a high BW and a high GA is significantly negative correlated with a low value of Direct Bilirubin. Another cause of neonatal jaundice is urinary tract infection (UTI). Shahian et al. (2012) found $12.5 \%$ of the asymptomatic jaundice neonates with the onset of unconjugated hyperbilirubinemia in the first week of life, and suggested that urine culture should be considered as a part of the diagnostic evaluation of jaundice neonates $>3$ days of life with an unexplained etiology (Shahian et al., 2012). On the other hand, Oswari et al. (2013) found that serum gamma-glutamyltransferase (GGT) and AST values can be used to predict the prognosis of patients with sepsis-associated cholestasis (Oswari et al., 2013). Our results show that there is a positive statistical correlation between BW, GA and GPT, a high BW or a high GA is correlated with a high GPT value. 
Table 3. Correlations between independent variables (1 min Apgar score, 5 min Apgar score, Gestational age, Birth weight) and biochemical parameters

\section{1 min Apgar score}

\begin{tabular}{l|c|c|c}
\hline & $\mathbf{r}^{\mathbf{a}}$ & Confidence interval $(\mathbf{9 5} \%)$ & $\boldsymbol{p}^{\mathbf{b}}$ \\
\hline Urea & -0.08372 & -0.3698 to 0.2169 & 0.5758 \\
\hline Creatinine & -0.3012 & -0.5407 to -0.01638 & $0.0336^{*}$ \\
\hline Total Bilirubin & 0.1412 & -0.1574 to 0.4161 & 0.3385 \\
\hline Direct Bilirubin & 0.02178 & -0.2889 to 0.3283 & 0.8897 \\
\hline GOT & 0.02761 & -0.2550 to 0.3058 & 0.8460 \\
\hline GPT & 0.2574 & -0.03110 to 0.5063 & 0.0712 \\
\hline CRP & -0.1415 & -0.4110 to 0.1508 & 0.3271 \\
\hline
\end{tabular}

5 min Apgar score

\begin{tabular}{l|c|c|c}
\hline & $\mathbf{r}^{\mathbf{a}}$ & Confidence interval (95\%) & $\boldsymbol{p}^{\mathbf{b}}$ \\
\hline Urea & -0.1393 & -0.4174 to 0.1626 & 0.3503 \\
\hline Creatinine & -0.2826 & -0.5262 to 0.003939 & $0.0468^{*}$ \\
\hline Total Bilirubin & 0.1140 & -0.1843 to 0.3930 & 0.4404 \\
\hline Direct Bilirubin & -0.1084 & -0.4037 to 0.2072 & 0.4888 \\
\hline GOT & 0.01448 & -0.2672 to 0.2939 & 0.9189 \\
\hline GPT & 0.2335 & -0.05643 to 0.4872 & 0.1026 \\
\hline CRP & -0.1801 & -0.4435 to 0.1118 & 0.2106 \\
\hline
\end{tabular}

Birth weight

\begin{tabular}{l|c|c|c}
\hline & $\mathbf{r}^{\mathbf{a}}$ & Confidence interval (95\%) & $\boldsymbol{p}^{\mathbf{b}}$ \\
\hline Urea & -0.2740 & -0.5266 to 0.02312 & 0.0624 \\
\hline Creatinine & -0.008219 & -0.2937 to 0.2786 & 0.9548 \\
\hline Total Bilirubin & -0.007929 & -0.2994 to 0.2849 & 0.9573 \\
\hline Direct Bilirubin & -0.5542 & -0.7369 to -0.2961 & $0.0001^{*}$ \\
\hline GOT & 0.09467 & -0.1910 to 0.3656 & 0.5044 \\
\hline GPT & 0.5324 & 0.2905 to 0.7104 & $0.0001^{*}$ \\
\hline CRP & -0.2504 & -0.5007 to 0.03856 & 0.0795 \\
\hline
\end{tabular}

\section{Gestational age}

\begin{tabular}{l|c|c|c}
\hline & $\mathbf{r}^{\mathbf{a}}$ & Confidence interval (95\%) & $\boldsymbol{p}^{\mathbf{b}}$ \\
\hline Urea & -0.2938 & -0.5420 to 0.001547 & $0.0450^{*}$ \\
\hline Creatinine & -0.06826 & -0.3477 to 0.2223 & 0.6376 \\
\hline Total Bilirubin & -0.01890 & -0.3093 to 0.2747 & 0.8985 \\
\hline Direct Bilirubin & -0.4443 & -0.6622 to -0.1571 & $0.0028^{*}$ \\
\hline GOT & 0.1060 & -0.1800 to 0.3754 & 0.4546 \\
\hline$G P T$ & 0.4558 & 0.1950 to 0.6563 & $0.0009^{*}$ \\
\hline CRP & -0.1143 & -0.3878 to 0.1777 & 0.4292 \\
\hline
\end{tabular}

Note: a - correlation coefficient; $\mathrm{b}$ - significance criterion $*$ - significant values where $p \leq 0.05$ 
CRP is an acute phase reactant, a protein synthesized and secreted by the liver in response to inflammatory cytokines, specifically IL-6 (Satar and Özlü, 2012) and is commonly used for bacterial sepsis detection in neonates. Still it is not useful as an early phase infection marker and it lacks specificity $(\mathrm{Ng}$ and Lam, 2006). All neonates in our study had a high CRP level, the mean being $8.21 \mathrm{mg} / \mathrm{dl}$. In their study, Zhou et al. (2016) have found a CRP level $>0.8 \mathrm{mg} / \mathrm{dl}$ in neonates $(39.1 \%)$ with positive blood culture results and $45.3 \%$ of them died within 7 days after birth, a higher prevalence than us (20.96\%) (Zhou et al., 2016). Also, Mannan et al. (2010) found that CRP was raised in $72 \%$ of cases of neonates with positive blood culture and only in $4 \%$ of control cases, and their study concluded that CRP is the most sensitive method (93\%) in culture proven sepsis, $79 \%$ in suspected sepsis and its positive predictive value in suspected sepsis amounts to $88 \%$.

Hofer et al. (2012) found that a growing body of evidence suggests a link between GA and CRP kinetics with lower baseline CRP values and a lower CRP response to infection in preterm compared to term newborns. All correlations between all independent variables that we studied (1 and 5 min Apgar score, GA, BW) and CRP are negatively correlated, a high value of independent variable is associated with a low CRP value, but it is not statistically significant. Hofer et al. (2012) conclude that CRP has the best diagnostic accuracy when combined with another infection marker like PCT, IL-6, and IL-8, that provides a higher sensitivity during the early phases of sepsis.

The gold standard for diagnostic sepsis is BC but the CRP is also particularly useful for monitoring the response to treatment and guiding antibiotic therapy. The highest level of CRP concentrations is detected during the first day of illness but because sustained proinflammatory action of IL-6, production could be detected until 24 hours after treatment was started. In their study, Janković et al. (2001) found that in the case of non-adequate initial antibiotic therapy of neonatal sepsis, CRP level increases further during the second day, but if the treatment is appropriate in the second day there is a significant decrease of CRP levels. CPR level can be taken as indication for replacement of initial antibiotics during the second day of treatment of sepsis neonates. The pathogens that are involved in neonatal sepsis are different depending on the type of neonatal sepsis, EOS or LOS, and the country's degree of development. Organisms associated with EOS are Group B Streptococcus (GBS, in special Streptococcus agalactiae), Escherichia coli $(E$. coli) which together account for about $70 \%$ of cases, and Streptococcus viridans. In LOS, organisms associated are CoNS, Staphylococcus aureus, Candida albicans and Klebsiella pneumoniae (Shah and Padbury, 2014; Cortese et al., 2016; Resende et al., 2015). In developed countries, in EOS are dominant GBS and E. coli, and in LOS are CoNS and GBS followed by Staphylococcus aureus (Hyde et al., 2002; Vergnano et al., 2005). In developing countries, the pathogens associated with EOS are E. coli, GBS, Enterobacter, Enterococcus, Listeria and with LOS Pseudomonas spp., Salmonella, Seratia. On both, EOS and LOS, are more associated Klebsiella, Acinetobacter, Staphylococcus aureus and also CoNS (Vergnano et al., 2005).

The appropriate empirical antibiotic selection during neonatal sepsis is based on the likely etiologic pathogens based on epidemiologic surveillance. Cortese et al. (2016) found that for EOS, the recommended empiric therapy as $1^{\text {st }}$ line is Ampicillin and an Aminoglycoside, and for LOS is Vancomycin and an Aminoglycoside. Also in our study the most used antibiotic was Aminoglycoside followed by Penicillin but for both of type of sepsis. 


\section{Conclusions}

There is a statistically significant negative correlation between 1 and 5 min Apgar score and creatinine, between Gestational Age and urea, and also between Birth Weight, Gestational Age and Direct Bilirubin. The statistically significant positive correlation is between Birth Weight, Gestational Age and alanine aminotransferase.

\section{References}

1. Agras PI, Tarcan A, Baskin E, Cengiz N, Gürakan B, Saatci U (2004) Acute renal failure in the neonatal period. Renal failure 26(3):305-309.

2. Bolat F, Comert S, Bolat G, Kucuk O, Can E, Bulbul A, Nuhoglu A (2013) Acute kidney injury in a single neonatal intensive care unit in Turkey. World Journal of Pediatrics 9(4):323-329.

3. Chand N, Sanyal AJ (2007) Sepsis-induced cholestasis. Hepatology 45(1):230-241.

4. Cortese F, Scicchitano P, Gesualdo M, Filaninno A, De Giorgi E, Schettini F, Ciccone MM (2016) Early and late infections in newborns: where do we stand? A review. Pediatrics \& Neonatology 57(4):265-273.

5. Hofer N, Zacharias E, Müller W, Resch B (2012) An update on the use of C-reactive protein in early-onset neonatal sepsis: current insights and new tasks. Neonatology 102(1):25-36.

6. Hug L, Alexander M, You D, Alkema L (2019) National, regional, and global levels and trends in neonatal mortality between 1990 and 2017, with scenario-based projections to 2030: a systematic analysis. The Lancet Global Health 7(6):e710-e720.

7. Hyde TB, Hilger TM, Reingold A, Farley MM, O'Brien KL, Schuchat A (2002) Trends in incidence and antimicrobial

\section{Conflict of Interest}

The authors declare that the research was conducted in the absence of any commercial or financial relationships that could be construed as a potential conflict of interest.

\section{Acknowledgments}

We want to thank Assoc. Prof. Edit Székely, M.D., PhD for her support in data collection.

resistance of early-onset sepsis: populationbased surveillance in San Francisco and Atlanta. Pediatrics 110(4):690-695.

8. Janković B, Pasić S, Marković M, Veljković D, Milicić M (2001) C-reactive protein concentrations during initial (empiric) treatment of neonatal sepsis. Srpski arhiv za celokupno lekarstvo 129:17-22.

9. Mannan MA, Shahidullah M, Noor MK, Islam F, Alo D, Begum NA (2010) Utility of C-reactive protein and hematological parameters in the detection of neonatal sepsis. Mymensingh medical journal: MMJ 19(2):259-263.

10. Mathur NB, Agarwal HS, Maria A (2006) Acute renal failure in neonatal sepsis. The Indian Journal of Pediatrics 73(6):499-502.

11. Newborns: reducing mortality (2018). In: World Health Organization site. https://www.who.int/en/news-room/factsheets/detail/newborns-reducing-mortality. Accessed 10 Jul 2019

12. Ng PC, Lam HS (2006) Diagnostic markers for neonatal sepsis. Current opinion in pediatrics 18(2):125-131.

13. Oswari H, Widjaja RK, Rohsiswatmo R, Cleghorn G (2013) Prognostic value of biochemical liver parameters in neonatal sepsis-associated cholestasis. Journal of paediatrics and child health 49(1):E6-E11. 
14. Resende DS, Peppe AL G, Reis HD, Abdallah VOS, Ribas RM, Gontijo Filho PP (2015) Late onset sepsis in newborn babies: epidemiology and effect of a bundle to prevent central line associated bloodstream infections in the neonatal intensive care unit. Brazilian Journal of Infectious Diseases 19(1):52-57.

15. Satar M, Özlü F (2012) Neonatal sepsis: a continuing disease burden. The Turkish journal of pediatrics 54(5):449.

16. Shah BA, Padbury JF (2014) Neonatal sepsis: an old problem with new insights. Virulence 5(1):170-178.

17. Shahian M, Rashtian P, Kalani M (2012) Unexplained neonatal jaundice as an early diagnostic sign of urinary tract infection. International Journal of Infectious Diseases 16(7):e487-e490.

18. Simonsen KA, Anderson-Berry AL, Delair SF, Davies HD (2014) Early-onset neonatal sepsis. Clinical microbiology reviews 27(1):21-47.

19. Vergnano S, Sharland M, Kazembe P, Mwansambo C, Heath PT (2005) Neonatal sepsis: an international perspective. Archives of Disease in Childhood-Fetal and Neonatal Edition 90(3):F220-FF224.

20. Whitehead MW, Hainsworth I, Kingham JGC (2001) The causes of obvious jaundice in South West Wales: perceptions versus reality. Gut 48(3):409-413.

21. Youssef D, Abd-Elrahman H, Shehab MM, Abd-Elrheem M (2015) Incidence of acute kidney injury in the neonatal intensive care unit. Saudi journal of kidney diseases and transplantation 26(1):67.

22. Zhou B, Liu X, Wu JB, Jin B, Zhang YY (2016) Clinical and microbiological profile of babies born with risk of neonatal sepsis. Experimental and therapeutic medicine 12(6): 621-3625. 